pulmonary physicians to better understand practise patterns and unmet needs in IPF.

Methods In December 2010 - January 2011, a structured, quantitative survey was conducted with lung specialists in IPF and nonIPF specialist centres principally in five EU countries (Germany, Italy, Spain, UK, France [5EU]). The survey was extended to a further five countries where fewer physicians were targeted. The survey covered clinical practise patterns over the preceding 12 months. Data from the UK are presented here.

Results The $5 \mathrm{EU}$ sample included 232 participating physicians. Of these, 26 were from the UK, with 15 from specialist IPF centres, and 11 from general lung clinics. Among the UK clinics, $46 \%$ of patients had stable IPF, versus $56 \%$ with progressive disease (51\% versus $49 \%$ respectively for $5 \mathrm{EU}$ ). Triple therapy (steroid + immunosuppressant $+\mathrm{N}$-acetylcysteine [NAC]) was prescribed for $31 \%$ of UK patients versus $25 \%$ for $5 \mathrm{EU}$. Nineteen percent of UK IPF patients went untreated, and a further $10 \%$ received palliative care. Steroid monotherapy was used in another $10 \%$ of UK patients, and $11 \%$ received steroid + immunosuppressant. NAC + steroids were used in only $5 \%$ of patients, and NAC alone in $6 \%$.

Conclusion IPF is a serious condition, but no standard therapy exists, and many patients receive no treatment or palliative care only. Current therapies are ineffective and unproven. At the time of the survey triple therapy remained prevalent; however, concerns have now been raised regarding AEs and risks of death and hospitalisation. There is therefore an unmet need for effective and welltolerated therapies to enable a greater range of patients to be treated with agents that have been proven to be safe and effective in clinical trials.

\section{P129 PATIENT AND PHYSICIAN DEMOGRAPHICS IN IDIOPATHIC PULMONARY FIBROSIS: RESULTS OF A UK PHYSICIAN SURVEY}

doi:10.1136/thoraxjnl-2012-202678.412

II Kausar, 'R Cameron, '2NC Eastmond. 'InterMune UK\&l, London, United Kingdom; 'Eastmond Medicomm Ltd, High Peak, United Kingdom

Introduction and Objectives Idiopathic pulmonary fibrosis (IPF) is a rare, progressive, fibrotic lung disorder of unknown origin, which leads to difficulty in breathing, functional disability and death. Due to symptomatic similarities with other lung conditions, IPF is difficult to diagnose, and patients experience varying standards of care. We undertook a survey of European pulmonologists to gain additional understanding of the characteristics of patients with IPF and the demographics of the physicians treating them.

Methods In December 2010-January 2011, a quantitative survey was conducted with lung specialists in IPF and non-IPF specialist centres in five principal EU countries (Germany, Italy, Spain, UK, France [5EU]). The survey was extended to a further five countries where fewer physicians were targeted. UK data are reported here.

Results The total number of patients seen by all the centres in the survey in the preceding 12 months was 7,302, 149 of which were from the UK. Among the UK patients, $87 \%$ were aged $\leq 85$ years, which is similar to the value for $5 \mathrm{EU}(85 \%)$. Sixty percent of newly diagnosed UK patients were aged $65-80$ years $(5 \mathrm{EU}=56 \%)$, in contrast to $50 \%$ of the whole UK sample (5EU $=57 \%$ ). In $5 \mathrm{EU}, 70 \%$ of IPF patients were managed by $35 \%$ of pulmonologists, in contrast to the UK where $80 \%$ of patients were managed by $55 \%$ of pulmonologists. In the UK, $70 \%$ of pulmonologists were seeing fewer than 110 IPF patients annually, whereas in the 5EU, 70\% of pulmonologists were seeing <30 IPF patients annually. Regional differences in the UK largely reflect population distribution with $14 \%$ of Scottish pulmonologists seeing $\geq 30$ IPF patients, versus $33 \%$ for the north of England, 24\% for the Midlands and Wales, and 29\% for the south of England.
Conclusion In the UK, patient demographics for IPF were similar to the rest of Europe, but UK patients were being diagnosed at a younger age than previously compared to the rest of the EU. Importantly, the UK had a much wider spread of IPF patients among treating pulmonologists than the rest of Europe, which indicates a greater distribution of expertise in treating the disease.

\section{P130 LIVING WITH IDIOPATHIC PULMONARY FIBROSIS: RESULTS OF A EUROPEAN PATIENT SURVEY}

doi:10.1136/thoraxjnl-2012-202678.413

C Giot, K Kirchgassler, M Maronati. InterMune International AG, Muttenz, Switzerland

Introduction and Objectives Idiopathic pulmonary fibrosis (IPF) is a rare, fibrotic, progressive lung disease that results in shortness of breath and difficulty in breathing. IPF commonly presents with crackling sounds in the lungs discernable via stethoscopic auscultation. IPF is most prevalent in patients $>50$ years of age. Risk factors include smoking and environmental exposures (metal, wood dust and other airborne pollutants). Disease progression features clubbing of the fingers, increasing difficulty in breathing, requirement for supplemental oxygen and/or lung transplantation. IPF is ultimately fatal. We conducted a European survey of diagnosed IPF patients to identify unmet needs in the management of IPF and opportunities to improve care.

Methods Patients in Germany, France, Italy, Spain and the UK with a confirmed diagnosis of IPF underwent a structured interview at home. In some instances, involved carers were also interviewed. The interviews featured associative questions where images were selected to express feelings, and constructive questions where factual recall was tested. Patient emotions were mapped according to four principle stages of the patient journey: 1. Symptoms, 2. Initial diagnosis, 3. After diagnosis, and 4. Disease management.

Results Forty-five patients (median age 67 years) and 18 care givers were interviewed for the survey. In $58 \%$ of cases, diagnosis was protracted due to dismissal of symptoms and misdiagnosis. Patients could be categorised in one of four ways (combative, serene, dejected and stoic) based chiefly on their age and attitude to disease management. Patients expressed a range of emotions at the point of diagnosis, and diagnosis was recognised as a time at which the patient-doctor relationship could break down. Patients treated in specialist centres reported better satisfaction with care than those treated by generalists. The patients cited negative impacts of IPF on almost all aspects of their lives, which could lead to depression. Keeping fit was acknowledged as a means to control disease symptoms and maintain a positive outlook.

Conclusion IPF has an enormous impact on patient quality of life, and patients are affected differently by the disease and its diagnosis. Emotional support and participation in patient support groups should be considered a key component of IPF management.

\section{P131 WHAT IS THE EVIDENCE FOR PHARMACOLOGICAL AND NON-PHARMACOLOGICAL INTERVENTIONS IN IMPROVING DYSPNOEA, OTHER SYMPTOMS AND QUALITY OF LIFE IN PROGRESSIVE IDIOPATHIC FIBROTIC INTERSTITIAL LUNG DISEASE?- A SYSTEMATIC REVIEW OF THE LITERATURE}

doi:10.1136/thoraxjnl-2012-202678.414

iS Bajwah, ${ }^{2} \mathrm{JR}$ Ross, ${ }^{3} \mathrm{JL}$ Peacock, ${ }^{4} \mathrm{~J}$ Higginson, ${ }^{5} \mathrm{AU}$ Wells, ${ }^{6} \mathrm{~A}$ Patel, ${ }^{4} \mathrm{~J} \mathrm{Koffman}$, 2J Riley. 'Department of Palliative Medicine, Royal MarsdenRoyal Brompton NHS Foundation Trusts, King's College London, Cicely Saunders Institute, Department of Palliative Care, Policy \& Rehabilitation, London, UK; ${ }^{2}$ Department of Palliative Medicine, Royal Marsden and Royal Brompton NHS Foundation Trusts \& National HeartLung Institute, Imperial College, London, UK; ${ }^{3}$ Division of Health and Social Care Research, King's College London, London, UK; 'King's College London, Cicely Saunders Institute, Department of Palliative Care, Policy \& Rehabilitation, London, UK; ${ }^{5}$ National HeartLung 